\title{
Minimizing the of shell and tube heat exchangers cost using evolutionary algorithms
}

\author{
${ }^{1}$ Dr.C. Maida Bárbara Reyes Rodríguez, ${ }^{1,3}$ Dr.C. Jorge Laureano Moya Rodríguez, \\ ${ }^{1}$ M.Sc. Eduardo Miguel Fírvida Donéstevez, ${ }^{2}$ Lic. Sergio R. Pérez León, ${ }^{3}$ Dr. Jandecy Cabral Leite \\ maidab@uclv.edu.cu,jorgemoyar@gmail.com, jandecy.cabral@itegam.org.br \\ ${ }^{1}$ Universidad Central Marta Abreu de Las Villas, UCLV, Cuba \\ ${ }^{2}$ Centro de Estudios Energéticos y de Tecnologías Ambientales (CEETA) \\ ${ }^{3}$ Instituto de Tecnología e Educación Galileo da Amazônia - ITEGAM
}

\begin{abstract}
Shell and Tube Heat exchangers (STHEs) are the most common type of heat exchangers. They are applied in numerous industrial installations. The minimization of the costs of these heat exchangers is a key goal for both designers and users. The design of this type of heat exchanger involves complex processes, including selection of the geometrical and operating parameters. The traditional design approach of Shell and Tube Heat Exchangers consists of the evaluation of a number of different exchanger geometries to identify those satisfying a requirement of heat and a set of geometric and operational constraints. However, this approach is slow, inaccurate and does not guarantee an optimal solution. In this paper, different evolutionary algorithms to optimize STHEs economically are analyzed and applied, including the particle swarm optimization, genetic algorithms and mixed ant colony optimization, this last one developed by the authors. As objective function the minimization of total annual cost was considered. For optimization were considered as independent design variables the inside diameter of the shell, the outer pipe diameter and spacing between the baffles. They were also considered for optimizing two patterns or arrangements of tubes, in a triangle and a square. An example of three case studies of optimization of heat exchanger is presented. The results of the optimization using the mixed ant colony technique are compared with those obtained using particle swarm and genetic algorithms.
\end{abstract}

Key words: Heat exchangers, optimization, evolutive algorithms, ant colony.

\section{Minimización del costo de los intercambiadores de calor de tubo y coraza mediante algoritmos evolutivos}

\section{RESUMEN}

Los intercambiadores de calor de tubo y coraza (ICTC) son el tipo más común de los intercambiadores de calor. Los mismos se aplican en numerosas instalaciones industriales. La minimización de los costes de estos intercambiadores de calor es un objetivo clave tanto para los diseñadores como para los usuarios. El diseño del intercambiador de calor implica procesos complejos, incluyendo la selección de los parámetros geométricos y parámetros de funcionamiento. El enfoque tradicional de diseño para los intercambiadores de calor de tubo y coraza consiste en la evaluación de un determinado número de geometrías diferentes del intercambiador para identificar aquellas que satisfacen un requerimiento de calor y un conjunto de restricciones geométricas y operacionales. Sin embargo, este enfoque es lento, poco preciso y no garantiza una solución óptima. En el presente trabajo se analizan y aplican diferentes algoritmos evolutivos para optimizar económicamente los mismos, entre ellos la optimización con enjambre de partículas, por algoritmos genéticos y por colonia de hormigas mixto, este último desarrollado por los autores. Como función objetivo se consideró la minimización del costo total anual. Para la optimización se consideraron como variables independientes de diseño el diámetro interior de la coraza, el diámetro exterior del tubo y el espaciamiento entre los deflectores. También se consideraron para la optimización dos disposiciones o arreglos de tubos: en forma de triángulo y en forma de cuadrado. Se presentan tres estudios de caso de la optimización de un intercambiador de calor. Los resultados de la optimización usando la técnica de colonia de hormigas mixta son comparados con aquellos obtenido usando enjambre de partículas y algoritmos genéticos.

Palabras Clave: Intercambiadores de Calor, optimización, algoritmos evolutivos,colonia de hormigas.

\section{INTRODUÇÃO}

La investigación y el desarrollo en la transferencia de calor son de significativa importancia en muchas ramas de la tecnología, en particular de la tecnología energética [1][2]. Los actuales desarrollos incluyen nuevos y eficientes intercambiadores de calor. Las áreas de aplicación incluyen la recuperación del calor en las industrias de procesos, en particular químicas y

ITEGAM - JETIA Vol. 01, No.04. Dezembro de 2015. Manaus - Amazonas, Brasil. ISSN 2447-0228 (ONLINE). 
petroquímicas que cada vez juegan un papel más creciente en Cuba y otros países. A través del mejoramiento sistemático de los diseños y la operación de los intercambiadores de calor se inserta la problemática del ahorro de energía, el desarrollo sostenible y la defensa y protección del medio ambiente, políticas que están aprobadas y constituyen planes y programas de investigación suscritos por la Academia de Ciencias de Cuba y el Ministerio de Ciencia, Tecnología y Medio Ambiente.

Los intercambiadores de calor (ICs) están presentes en la mayoría de los sistemas térmicos complejos de las industrias y representan el vehículo más ampliamente usado para la transferencia de calor en las aplicaciones de los procesos industriales. Son seleccionados para servicios tales como: enfriamiento de líquido o gas, procesos donde se condensen vapores de refrigerantes o vapor de agua, procesos de evaporación de refrigerantes agua u otros líquidos; procesos de calentamiento regenerativo del agua de alimentación a calderas, para la recuperación del calor en efluentes gaseosos y líquidos residuales calientes, para el enfriamiento de aire y aceite de lubricación en compresores, turbinas y motores, mediante camisas de enfriamiento y muchas otras aplicaciones industriales [3].

Los ICs tienen la habilidad de transferir grandes cantidades de calor con relativamente bajo costo, poseyendo grandes áreas de superficie de transferencia en pequeños espacios, volúmenes de líquido y peso. En la figura 1 se muestra un esquema simplificado de un IC, en particular, de tubo y coraza [4].

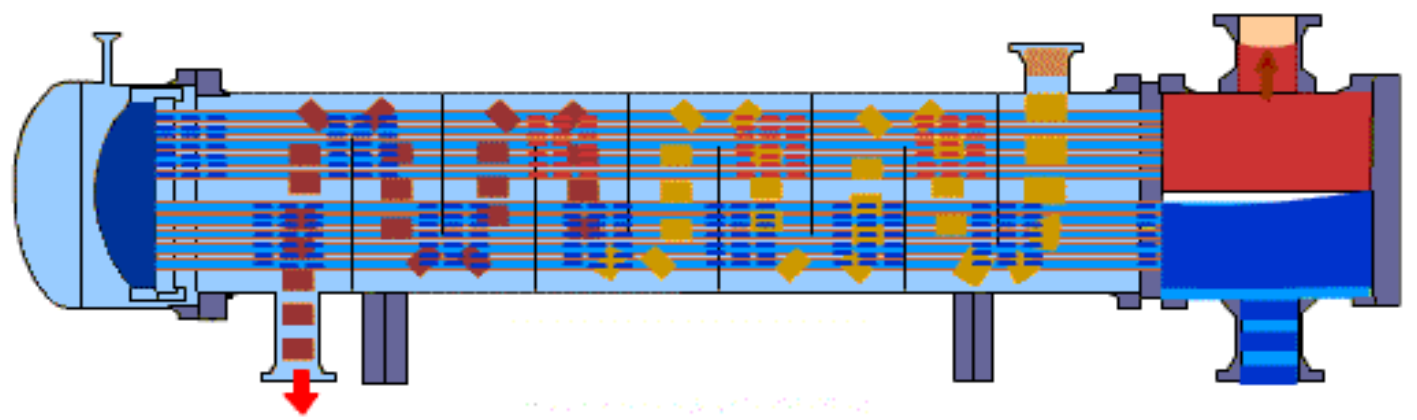

Figura 1. Esquema de un intercambiador de calor de tubo y coraza.

\section{MÉTODOS PARA EL DISEÑO DE LOS INTERCAMB IADORES DE CALOR DE TUBO Y CORAZA.}

La dificultad para integrar las correlaciones obtenidas en los bancos de tubos y el flujo generado en la carcasa de un intercambiador con deflectores impulsó el desarrollo de "métodos integrales" para el cálculo de la transferencia de calor y pérdida de carga en el lado carcasa. Entre ellos se pueden citar los siguientes: Método de Tinker (años 50), Método de Kern (años 50), Método de Bell-Delaware (años 60), Método de PalenTaborek (años 60-70), Método de Wills and Jhonston. (años 7080).

En los últimos años los algoritmos poblacionales y de inteligencia colectiva de optimización han Ganado popularidad y son utilizados en varias áreas del conocimiento, debido a su relativa simplicidad y costo computacional. Estos algoritmos se están empleando en la optimización de intercambiadores de calor de Tubo y Coraza, debido a que son capaces de encontrar buenas soluciones con un mínimo de esfuerzo; convergiendo hacia un único óptimo global.

\section{APLICACIÓN DE DIFERENTES MÉTODOS DE LA INTELIGENCIA ARTIFICIAL A LA OPTIMIZACIÓN DEL COSTO DE LOS \\ INTERCAMBIADORES DE CALOR DE TUBO Y CORAZA.}

Hay muchos estudios previos sobre la optimización de intercambiadores de calor. Varios investigadores han utilizado diferentes técnicas de optimización considerando diferentes funciones objetivo para optimizar el diseño del intercambiador de calor. En [5] usaron el enfoque del recocido simulado para el diseño óptimo de intercambiadores de calor y desarrollaron un procedimiento de comandos para enlazar el programa HTRI (Heat Transfer Research Inc.) con el algoritmo del recocido. Los autores habían analizado el problema, considerando dos funciones objetivos diferentes llamadas, el área total de transferencia de calor y un coste índice de costo lineal obtenido.

En [6] usaron el algoritmo genético (GA) para el diseño óptimo de STHEs (intercambiadores de calor de tubo y coraza), en el que la caída de presión se aplicó como una restricción para lograr los parámetros óptimos de diseño. Los autores habían considerado la minimización del costo total del intercambiador de calor como una función objetivo. Un estudio de caso fue considerado también para demostrar la aplicación del algoritmo. Par [7] consideró un problema mixto de programación no lineal de STHE teniendo en cuenta el tamaño y el costo de la exergía del STHE. Los autores habían considerado la minimización de la suma del costo anual del capital y el coste exergético de STHEs como una función objetivo y aplicaron un algoritmo genético para la optimización.

En [8] llevaron a cabo el diseño del intercambiador de calor basados en optimización económica usando GA (algoritmos genéticos). Los autores habían considerado la minimización del costo total anual como una función objetivo para encontrar la óptima arquitectura del intercambiador de calor.

Para [9] usaron el análisis de sensibilidad global para identificar los parámetros geométricos más influyentes que afectan el costo total de los STHE con el fin de reducir el tamaño del problema de optimización y llevar a cabo la optimización de los parámetros influyentes de los SYHE desde el punto de vista económico mediante la aplicación del algoritmo de búsqueda armónico. Los autores habían considerado la minimización de los costos de capital y los costos de operación como una función objetivo. 
Lo autor [10] explora el uso de una técnica de optimización no tradicional, llamada Optimización por enjambre de partículas (PSO), para la optimización del diseño de los intercambiadores de calor de tubo y coraza, desde el punto de vista económico. La minimización del costo total anual se considera como función objetivo. Tres variables de diseño tales como el diámetro interior de la coraza, el diámetro exterior del tubo y el espaciamiento entre deflectores se consideran para la optimización. También se consideraron para la optimización dos disposiciones de tubos en forma de triángulo y en forma de cuadrado.

Varios otros investigadores también usaron estrategias basadas en GA (algoritmos genéticos) y algoritmos tradicionales de optimización matemática [11][12][13][14][15][16][17][18][19][20][21][22][23][24][25][2 6][27] para diversos objetivos como la generación de entropía mínima [11][17] y el costo mínimo de STHEs [12][13][15][18][20][21][22][24][25][26][27] para optimizar el diseño de intercambiador es de calor. Algunos de estos estudios se focalizan en un solo parámetro geométrico como el espaciamiento óptimo del deflector [13][14][15] y algunos otros tratan de optimizar una gran variedad de parámetros geométricos y operativos de los STHEs. Sin embargo, hay una necesidad de investigar el potencial de la aplicación de técnicas de optimización no tradicionales.

En el presente trabajo se comparan los resultados de la optimización del costo de los intercambiadores de calor de tubo y coraza a través de los Algoritmos Genéticos, el Enjambre de Partículas (Particle Swarm Optimization) (PSO) y mediante Colonia de hormigas mixta (Mixed Ant Colony Optimization) (MACO) que fue desarrollado por los autores para optimizar intercambiadores de calor.

\section{MODELO MATEMÁTICO PARA EL DISEÑO Y C ÁLCULO DE UN INTERCAMBIADOR DE TUBO Y CORA ZA (MÉTODO KERN).}

A continuación se presentarán una serie de ecuaciones que se combinarán para bosquejar la solución de un intercambiador de calor de carcasa y tubo.

Para [28] desarrolló uno de los primeros métodos para el estudio del comportamiento térmico de un intercambiador de calor de tubo y coraza. Kern a partir de un intercambiador estándar restringido para un corte de las mamparas del 25\% (el mejor en la mayoría de los casos) obtiene las correlaciones para el cálculo de transferencia de calor y la pérdida de cargas.

El cálculo se realizará por el método Kern, que todavía se sigue utilizando en la industria para comprobar el funcionamiento térmico de los intercambiadores. Este considera que el flujo a través del arreglo de tubos es únicamente flujo cruzado, y no contempla fugas entre los componentes de la coraza.

Antes de comenzar el cálculo, las condiciones de proceso requeri das para ambos fluidos son:

- Temperaturas de entrada y salida $\left(T_{\mathbb{i}}, T_{\bullet}\right)$.

- Flujos másicos $(m)$.

- Capacidades caloríficas $(C p)$.

- Conductividades térmicas $(\lambda)$.

- Densidades $(\rho)$.
- Viscosidades $(\mu)$.

- Factores de obstrucción o ensuciamiento (Rensuc).

- Número de años de vida de la instalación (ny).

- Tasa de descuento anual (i) .

- Costo de la energía eléctrica $\left(C_{E}\right)$.

- Horas de trabajo al año $(H)$.

Las variables a optimizar serian.

- Diámetro exterior del tubo (do).

- Diámetro interior de la coraza $\left(D_{s}\right)$.

- Número de pases de los tubos $(N p)$.

- Espaciamiento entre bafles $(B)$.

- Tipo de distribución.

Teniendo en cuenta el coeficiente global de transferencia de calor, el área de la superficie del intercambiador de calor $\left(A_{T}\right)$ se calcula:

$$
A_{T}=\frac{Q}{U * F * L M T D}
$$

Para la transferencia de calor sensible, la tasa de transferencia de calor está dada por,

$$
\mathrm{Q}=m_{\mathrm{h}} * \mathrm{C}_{\mathrm{ph}}\left(\mathrm{T}_{\mathrm{hi}}-\mathrm{T}_{\mathrm{hQ}}\right)=m_{e} * \mathrm{C}_{\mathrm{pc}}\left(\mathrm{T}_{\mathrm{co}}-\mathrm{T}_{\mathrm{ci}}\right)
$$

Donde $T_{c i}$ es Temperatura de entrada del líquido frío en ${ }^{\circ} C, T_{c o}$, Temperatura de salida del líquido frío en ${ }^{\circ} C, T_{\text {hi }}$, Temperatura de entrada del líquido caliente en ${ }^{\circ} \mathrm{C}, T_{\mathrm{h}}$, Temperatura de salida del líquido caliente en ${ }^{\circ} C, C_{\text {ph }}$, Calor específico del líquido caliente en $\mathrm{kJ} / \mathrm{kg}^{\circ} \mathrm{C}, C_{\text {pe }}$, Calor específico del líquido frío en $\mathrm{kJ} / \mathrm{kg}^{\circ} \mathrm{C}, \mathrm{m}_{\varepsilon}$, flujo másico del fluido frío en $\mathrm{kg} / \mathrm{seg}, \mathrm{m}_{\mathrm{h}}$, flujo másico del fluido caliente en $\mathrm{kg} / \mathrm{seg}$.

Teniendo en cuenta el flujo cruzado entre el deflector adyacente, diferencia de temperatura media logarítmica (LMTD) se determina por,

$$
L M T D=\frac{\left(T_{h i}-T_{c c}\right)-\left(T_{h o}-T_{c i}\right)}{\ln \left(\frac{T_{h i}-T_{c o}}{T_{h o}-T_{c i}}\right)}
$$

El factor de corrección $F_{t}$ para la configuración de flujo en cuestión se encuentra en función de la relación de temperatura adimensional para la mayoría de las configuraciones de flujo de interés [29[30].

$$
F_{t}=\frac{\sqrt{R^{2}+1}}{R-1} * \frac{\operatorname{In}\left(\frac{1-P}{1-F R}\right)}{\operatorname{In} \frac{\left(2-F\left(R+1-\sqrt{R^{2}+1}\right)\right]}{\left[2-P\left(R+1+\sqrt{R^{2}+1}\right)\right]}}
$$

donde $\mathrm{R}$ es el coeficiente de corrección dado por:

$$
\mathrm{R}=\frac{\mathrm{T}_{\mathrm{hi}}-\mathrm{T}_{\mathrm{hq}}}{\mathrm{T}_{\mathrm{cc}}-\mathrm{T}_{\mathrm{ci}}}
$$

P es otro coeficiente, dado por:

$$
P=\frac{T_{c o}-T_{c i}}{T_{h i}-T_{c i}}
$$


Maida Bárbara Reyes Rodríguez et al./ ITEGAM-JETIA Vol.01, No 04, pp.09-18. Dezembro, 2015.

El coeficiente global de transferencia de calor $(U)$ depende de los coeficientes de transferencia de calor y las resistencias al ensuciamiento tanto del lado del tubo como del lado de la coraza dado por [31]:

$$
U=\frac{1}{\frac{1}{h_{s}}+\text { Renswes }+\frac{d_{g}}{d_{i}}\left(\text { Renswet }+\frac{1}{h_{\mathrm{L}}}\right)}
$$

Donde $d_{i}$ es el diametro interno del tubo y esta dado por,

$$
d_{\mathrm{i}}=0,8 * d_{0}
$$

$d_{0}$ es una de las variables independientes.

\section{IV.1 LADO DEL TUBO}

De acuerdo con el régimen de flujo, el coeficiente de transferencia de calor en el lado del tubo $\left(h_{\mathrm{t}}\right)$ se calcula a partir de la siguiente correlación $\mathrm{Si}\left(R e_{\mathrm{t}}<2300 ;\right.$ [32],

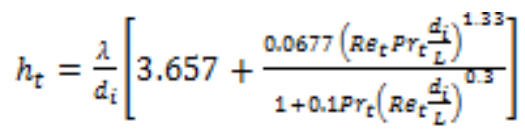

$\operatorname{Si}\left(2300<R e_{\mathrm{t}}<10000 ;[32]\right.$,

$$
h_{t}=\frac{\Omega}{d_{i}}\left\{\frac{f_{t}\left(R \varepsilon_{t}-1000\right) P v_{t}}{1+12.7 \sqrt{\frac{f_{t}}{g}\left(P v_{t}^{0.67}-1\right)}}\left[1+\left(\frac{d_{i}}{L}\right)^{0.67}\right]\right\}
$$

$\operatorname{Si}\left(R e_{\mathrm{t}}>10000 ;[32]\right.$,

$$
h_{\mathrm{t}}=\frac{\pi}{d_{0}} 0.027 \operatorname{Re}_{\mathrm{t}}^{0.8} \operatorname{Pr}_{\mathrm{t}}^{\frac{1}{3}}\left(\frac{\mu_{\mathrm{t}}}{\mu_{\mathrm{g}}}\right)^{0.14}
$$

Donde $f_{t}$ es el factor de friccion de Darcy [33] dado por:

$$
f_{t}=\left(1.92 \log _{10} R e_{t}-1.64\right)^{-2}
$$

$R e_{t}$ es el número de Reynolds en la cara del tubo y está dado como:

$$
R e_{t}=\frac{\rho w_{t} d_{i}}{\mu}
$$

Velocidad de flujo para el lado del tubo se encuentra por,

$$
v_{t}=\frac{G_{x}}{\rho}
$$

Donde la velocidad másica del agua está dada por,

$$
\mathrm{G}_{\mathrm{s}}=\frac{\mathrm{m}_{\mathrm{t}}}{A_{\mathrm{t}}}
$$

Donde el área total de flujo es,

$$
A_{\mathrm{t}}=A_{\mathrm{gtt}} * \frac{N_{t}}{N_{D}}
$$

Donde $N_{p}$ es el número de pases por los tubos (otra variable independiente) y $A_{\text {gtt }}$ es el área de la sección transversal del tubo está dada por:

$$
A_{s t t}=\frac{\pi}{4} * d_{i}^{2}
$$

$N_{t}$ es el número de tubos y n es el número de pasos por los tubos que puede ser hallado aproximadamente por la siguiente ecuación.

$$
N_{t}=C\left(\frac{D_{s}}{d_{0}}\right)^{n_{1}}
$$

C y $n_{1}$ son coeficientes que están tomando valores de acuerdo con la disposición de flujo y el número de pasadas.

Tabla 1. Valores de los coeficientes $C$ y $n_{1}$.

\begin{tabular}{|c|c|c|c|c|}
\hline \multirow{2}{*}{ No. De pases (NP) } & \multicolumn{2}{|c|}{ Arreglo de tubos triangular } & \multicolumn{2}{c|}{ Arreglo de tubos cuadrado } \\
\cline { 2 - 5 } & $\mathrm{C}$ & $\mathrm{n} 1$ & $\mathrm{C}$ & $\mathrm{n} 1$ \\
\hline 1 & 0,319 & 2,142 & 0,215 & 2,207 \\
\hline 2 & 0,249 & 2,207 & 0,156 & 2,2291 \\
\hline 4 & 0,175 & 2,285 & 0,158 & 2,263 \\
\hline 6 & 0,0743 & 2,499 & 0,0402 & 2,617 \\
\hline 8 & 0,0365 & 2,675 & 0,0331 & 2,643 \\
\hline
\end{tabular}

Fuente: [34].

Sobre la base de la superficie total del intercambiador de calor (A), la longitud del tubo necesaria (L) es,

$$
\mathrm{L}=\frac{\mathrm{A}_{T}}{\pi \mathrm{d}_{0} \mathbb{N}_{\mathrm{t}}}
$$

Prt es el número de Prandtl en el lado del tubo y está dado por,

$$
\operatorname{Pr}=\frac{c_{p} \cdot d}{x}
$$

Donde $\lambda$ es la conductividad térmica del fluido (dato). 


\section{IV.2 LADO DE LA CORAZA}

La Formulación de Kern para intercambiadores de calor de tubo y coraza de separadores segmentados se usa para calcular el coeficiente de transferencia de calor en el lado de la coraza hs [34],

$$
\mathrm{h}_{s}=\left(0,36 \mathrm{R}_{\mathrm{es}}^{0,55} \cdot \mathrm{P}_{\mathrm{rs}}{ }^{1 / \mathrm{a}} \cdot\left(\frac{\mathrm{p}}{\mathrm{p}_{\mathrm{W}}}\right)^{0.14}\right) \frac{\lambda}{\mathrm{D}_{\mathrm{e}}}
$$

donde, De es el diámetro hidráulico de la coraza y se calcula como [32][34]:

Para distribución cuadrada:

$$
\mathrm{D}_{\mathrm{e}}=4 \cdot\left(\frac{\mathrm{p}_{\mathrm{t}}^{2}-\frac{\pi \mathrm{d}_{\mathrm{n}}^{2}}{4}}{\pi \mathrm{d}_{0}}\right)=\frac{1,27}{\mathrm{~d}_{0}}\left(\mathrm{P}_{\mathrm{t}}^{2}-0,785 . \mathrm{d}_{0}^{2}\right)
$$

Para distribución triangular equilátera:

$$
\mathrm{D}_{\mathrm{e}}=\frac{1,10}{\mathrm{~d}_{0}}\left(\mathrm{P}_{\mathrm{t}}^{2}-0,917 \cdot \mathrm{d}_{0}^{2}\right)
$$

Donde el paso entre los tubos se calcula como:

$$
P_{t}=(1.25 \div 1.5) \cdot d_{0}
$$

El número de Reynolds para el lado de la coraza es:

$$
\operatorname{Re}=\frac{\rho w_{g} \cdot D_{s}}{p}
$$

El número de Prandtl para el lado de la coraza es:

$$
\operatorname{Pr}=\frac{C_{D}, H}{\lambda}
$$

La velocidad de flujo para el lado de la coraza se puede obtener a partir de [31].

$$
\begin{aligned}
& v_{S}=\frac{G_{5}}{p} \\
& G_{S}=\frac{G}{A_{5}}
\end{aligned}
$$

$\mathrm{G}=m_{h}$ es el flujo másico del fluido que va por la coraza (dato) El área de la sección transversal normal a la dirección del flujo se determina por [31],

$$
A_{S=}=\frac{D_{s} \cdot B\left(P_{t}-d_{0}\right)}{F_{t}}=D_{s} B\left(1-\frac{d_{0}}{F_{t}}\right)
$$

Donde $\mathrm{D}_{\mathrm{s}}$ es diámetro interior de la coraza y $\mathrm{B}$ el espaciamiento entre bafles, ambos variables independientes del problema.

\section{FUNCIÓN OBJETIVO: MINIMIZACIÓN DEL COST O DE INVERSIÓN Y DE OPERACIÓN}

El costo total $C_{\text {tot }}$ se toma como la función objetivo, que incluye inversión de capital $\left(C_{\overline{\mathrm{i}}}\right)$, el costo de la energía $\left(C_{E}\right)$, el costo de operación anual $\left(C_{0}\right)$ y el costo total de descuento de operación de $\left(C_{O D}\right)[8]$.

$$
C_{\text {tot }}=C_{\text {inversion }}+C_{\text {operación }}
$$

Adoptando la correlación de Hall [35], el capital de inversión Ci se calcula en función del área superficial del intercambiador.

$$
C_{\text {inversion }}=a_{1}+a_{2} A_{T}^{a_{3}}
$$

Donde para intercambiadores de calor de acero inoxidable, tanto los tubos como la coraza [35] $a_{1}=8000, a_{2}=259,2$ y $a_{a}=0,91$.

El costo de operación relacionado con la potencia consumida en el bombeo para vencer las pérdidas friccionales se determina de las siguientes expresiones:

$$
C_{\text {operacion }}=\sum_{k=1}^{n y} \frac{c_{o}}{\left(1+D^{k}\right.}
$$

$$
C_{0}=P C_{E} H
$$

$C_{E}$ es el costo de la energía eléctrica en $\$ / \mathrm{kW}$ h, i la tasa de descuento anual, ny el número de años de vida de la instalación, $H$ las horas de trabajo al año.

$$
P=\frac{1}{\eta}\left(\frac{m_{t}}{\rho_{t}} \Delta P_{t}+\frac{m_{s}}{\rho_{s}} \Delta P_{s}\right)
$$

Donde $\eta$ es la eficiencia de bombeo (entre 0,5 y 0,7 ), $m_{t}$ y $m_{g}$ son los flujos másicos en el tubo y la coraza, $\Delta P_{t}$ es la caida de presión en los tubos, $\Delta P_{\Omega}$ es la caída de presión en la coraza, $P_{t}$ es la densidad del fluido que va por el tubo, $\rho_{s}$ es la densidad del fluido que va por la coraza

La caída de presión permisible en el intercambiador de calor es la presión estática del fluido que puede ser invertida para conducir el fluido a través del intercambiador. En todos los intercambiadores de calor hay una cerrada afinidad física y económica entre la transferencia de calor y la caída de presión. Para una capacidad calorífica constante en el intercambiador de calor que se va a diseñar, el incremento de la velocidad del flujo provocará un aumento del coeficiente de transferencia de calor lo que se traduce en el diseño compacto del intercambiador y un menor costo de inversión.

Sin embargo, aumentar la velocidad de flujo causará una mayor caída de presión en el intercambiador de calor lo que resulta en costes de funcionamiento adicional. Por esta razón cuando se diseña un intercambiador de calor la caída de presión debe ser considerada con la transferencia de calor y debe ser encontrada la mejor solución para el sistema.

La caída de presión del lado del tubo incluye la caída de presión distribuida a lo largo de la longitud del tubo y las pérdidas de presión concentradas en los codos y en las boquillas de entrada y salida [34].

La caída de presión en los tubos se calcula como la caída de presión propiamente dicha en los tubos más la caída de presión en los codos.

$$
\Delta P_{\mathrm{t}}=\Delta P_{\mathrm{lt}}+\Delta P_{\mathrm{cod}}
$$




$$
\Delta P_{t}=\frac{\rho_{t} v_{t}^{*}}{2}\left(\frac{L}{d_{i}} f_{t}+p\right) N_{p}
$$

donde,

$$
f_{t}=0.00128+0.1143\left(R e_{\mathrm{t}}\right)^{-0.211}
$$

Diferentes valores de la constante $p$ son considerados por diferentes autores. Kern [34] supone $p=4$, mientras que 32] suponen $p=2,5$. Se debe asumir 2,5, $N_{t}$ es el número de tubos (ya calculada), $L$ la longitud de los tubos (ya calculada), $v_{t}$ la velocidad del fluido en el lado del tubo (ya calculada).

La caída de presión del lado de la coraza es,

$$
\Delta P_{g}=\frac{p_{s} W_{s}^{2}}{2} f_{g} \frac{L}{B} \frac{D_{s}}{D_{g}}
$$

Donde el factor de fricción en el lado de la coraza se calcula como:

$$
f_{S}=1,44 R e_{g}^{-0,15}
$$

\section{ANÁLISIS DE RESULTADOS. ESTÚDIOS DE CASOS}

La eficacia de los diferentes métodos se evalúa mediante el análisis de tres estudios de caso:

Caso 1: 4,34 (MW) de necesidad, intercambiador de agua salobre - metanol [32].

Caso 2: 1,44 (MW) de necesidad, intercambiador de petróleo crudo - kerosene [34].

Caso 3: 0,46 (MW) de necesidad, intercambiador de agua destilada - agua cruda [32].

Los tres casos de estudio fueron analizados por [10] utilizando PSO, (A.C. Caputo 2008) utilizando el enfoque de los GA y tomados de la literatura [32][34].

Las especificaciones de diseño original, mostradas en las tablas, son suministradas como entrada al algoritmo MACO (Optimización por Colonia de Hormigas Mixtos). Las arquitecturas resultantes de intercambiadores óptimos obtenidas por MACO se comparan con los resultados obtenidos por [10] utilizando PSO, por [8] utilizando el enfoque GA y con la solución de diseño original dada por Sinnot [32] y Kern [34] (mostradas en las tablas).

A fin de permitir una comparación coherente, las funciones costo de los cuatros enfoques se calculan como se describe en la sección 5. También por la misma razón, todos los valores relacionados con los costos se han tomado del trabajo de [8] que trató todos los casos de estudios mediante enfoque por Algoritmos Genéticos.

Para el análisis se impusieron los siguientes límites superior e inferior para las variables de optimización:
- Diámetro interior de la coraza $\left(D_{s}\right)$ en un rango entre $0,1 \mathrm{~m}$ y $1,5 \mathrm{~m}$,

- Diámetro exterior de los tubos (do) en un rango entre los $0.015 \mathrm{~m}$ y $0.051 \mathrm{~m}$,

- Espaciamiento entre los deflectores $(B)$, en un rango de $0,05 \mathrm{~m}$ a $0,5 \mathrm{~m}$.

- Patrón de diseño de 30 y 45 grados.

- Número de pases de tubo $(N p)$ de 1, 2, 4, 6 y 8.

Todos los valores de los costos de descuento de operación se calculan con:

- Número de años ny = 10 años.

- Tasa de descuento anual $(i)=10 \%$.

- Costo de la energía $\left(C_{E}\right)=0,12 \mathrm{~h}$ Euros / kW.

- Cantidad de horas de trabajo anual $(H)=7000$ años/horas.

Para obtener la solución óptima usando MACO, se usaron como parámetros:

- $\quad$ Número de hormigas $=100$

- Número máximo de iteraciones $=1000$

Tabla 2. Propiedades físicas de entrada del caso1.

\begin{tabular}{|l||c||r||r|}
\hline \multicolumn{2}{|l||}{} & Coraza & \multicolumn{1}{c|}{ Tubo } \\
\hline \multicolumn{1}{|l||}{} & \multicolumn{1}{|c||}{ Metanol } & Agua de mar \\
\hline Flujo másico & $T_{i}$ & 95 & 68,9 \\
\hline Temperatura de entrada & $T_{o}$ & 40 & 25 \\
\hline Temperatura de salida & $\rho$ & 750 & 40 \\
\hline Densidad del fluido & $C p$ & 2,84 & 995 \\
\hline Calor especifico & $\mu$ & 0,00034 & 4,2 \\
\hline Viscosidad del fluido & $\lambda$ & 0,19 & 0,0008 \\
\hline Conductividad térmica & $\lambda$ & 0,59 \\
\hline Resistencia de ensuciamiento & Rensuc & 0,00033 & 0,0002 \\
\hline
\end{tabular}

Fuente: [32].

En los resultados mostrados en la Tabla 3 se puede notar que el diseño obtenido con el algoritmo MACO oferece

valores menores del coste total anual.

La principal diferencia entre los resultados es en la caída de presión en los tubos, lo que eleva los costos de bombeo necesarios para el intercambiador. Nuestra solució $\mathrm{n}$ muestra una reducción en costo anual de operación y el costo total de operación de descuento, determina do por una reducción de la caída de presión en los tubos. Las grandes diferencias en coeficientes de películ a están vinculados a un uso ineficiente de las caídas de presión. 
Maida Bárbara Reyes Rodríguez et al./ ITEGAM-JETIA Vol.01, No 04, pp.09-18. Dezembro, 2015.

Tabla 3. Geometrías Optimas del intercambiador de calor caso 1 usando diferentes métodos.

\begin{tabular}{|l|r|r|r|r|}
\hline & Literatura & \multicolumn{1}{c|}{ GA } & \multicolumn{1}{c|}{ PSO } & \multicolumn{1}{c|}{ MACO } \\
\hline L & 4,83 & 3,379 & 3,115 & 2,994 \\
\hline do & 0,02 & 0,016 & 0,015 & 0,015 \\
\hline B & 0,356 & 0,5 & 0,424 & 0,5 \\
\hline Ds & 0,894 & 0,83 & 0,81 & 0,84 \\
\hline Pt & 0,025 & 0,02 & 0,0187 & 0,0187 \\
\hline $\mathrm{Nt}$ & 918 & 1567 & 16558 & 1829 \\
\hline vt & 0,75 & 0,69 & 0,67 & 0,66 \\
\hline Ret & 14925 & 10936 & 10503 & 9997 \\
\hline Prt & 5,7 & 5,7 & 5,7 & 5,69 \\
\hline ht & 3812 & 3762 & 3721 & 3672 \\
\hline ft & 0,028 & 0,031 & 0,0311 & 0,0314 \\
\hline$\Delta$ Pt & 6251 & 4298 & 4171 & 1985 \\
\hline As & 0,032 & 0,0831 & 0,0687 & 0,084 \\
\hline De & 0,014 & 0,011 & 0,0107 & 0,0106 \\
\hline vs & 0,58 & 0,44 & 0,53 & 0,43 \\
\hline Res & 18381 & 11075 & 12678 & 10282 \\
\hline Prs & 5,1 & 5,1 & 5,1 & 5,08 \\
\hline hs & 1573 & 1740 & 1950,8 & 1767,3 \\
\hline fs & 0,33 & 0,357 & 0,349 & 0,36 \\
\hline$\Delta$ Ps & 35789 & 13265 & 20551 & 12322 \\
\hline U & 615 & 660 & 713,9 & 672,8 \\
\hline A & 378,6 & 262,8 & 243,2 & 258 \\
\hline Ci & 51507 & 49259 & 46453 & 48583 \\
\hline Co & 2111 & 947 & 1038,7 & 713 \\
\hline Cod & 12973 & 5818 & 6778,2 & 4381,3 \\
\hline Ctot & 64480 & 55077 & 53231,1 & 52964 \\
\hline
\end{tabular}

Fuente: Los autores.

VI.2 CASO 2: INTERCAMBIADOR DE PETRÓLEO CRUDO

- KEROSENE [34].

Caso 2. Este ejemplo implica dos fluidos con las propiedades que se muestran en la tabla 4 . Un resumen de los resultados obtenidos con el modelo propuesto se dan en la Tabla 5, donde se ofrece una comparación con los resultados reportados por [10] utilizando PSO, con [8] utilizando el enfoque GA y con la solución de diseño original dada por Sinnot [32] y Kern [34].

Tabla 4. Propiedades físicas de entrada del caso 2.

\begin{tabular}{|l|r|r|r|}
\hline \multirow{2}{*}{} & & \multicolumn{2}{|c|}{ CASO 2 } \\
\cline { 3 - 4 } & & Coraza & \multicolumn{1}{c|}{ Tubo } \\
\cline { 3 - 4 } & & Kerosene & Petróleo crudo \\
\hline Flujo másico & $m$ & 5,52 & 18,8 \\
\hline Temperatura de entrada & $T_{i}$ & 199 & 37,8 \\
\hline Temperatura de salida & $T_{o}$ & 93,3 & 76,7 \\
\hline Densidad del fluido & $\rho$ & 850 & 995 \\
\hline Calor especifico & $C p$ & 2,47 & 2,05 \\
\hline Viscosidad del fluido & $\mu$ & 0,0004 & 0,00358 \\
\hline Conductividad térmica & $\lambda$ & 0,13 & 0,13 \\
\hline Resistencia de ensuciamiento & Rensuc & 0,00061 & 0,00061 \\
\hline
\end{tabular}

Fuente: [34]. 
Maida Bárbara Reyes Rodríguez et al./ ITEGAM-JETIA Vol.01, No 04, pp.09-18. Dezembro, 2015.

Tabla 5. Geometrías Óptimas del intercambiador de calor del caso 2 usando diferentes métodos.

\begin{tabular}{|l|r|r|r|r|}
\hline & Literatura & \multicolumn{1}{|c|}{ GA } & \multicolumn{1}{c|}{ PSO } & \multicolumn{1}{c|}{ MACO } \\
\hline L & 4,88 & 2,153 & 1,56 & 6,41 \\
\hline do & 0,025 & 0,02 & 0,015 & 0,015 \\
\hline B & 0,127 & 0,12 & 0,1112 & 0,29 \\
\hline Ds & 0,539 & 0,63 & 0,59 & 0,33 \\
\hline Pt & 0,031 & 0,025 & 0,0187 & 0,0187 \\
\hline Nt & 158 & 391 & 646 & 231 \\
\hline vt & 1,44 & 0,87 & 0,93 & 1,44 \\
\hline Ret & 8227 & 4068 & 3283 & 4845 \\
\hline Prt & 55,2 & 55,2 & 55,2 & 56,4 \\
\hline ht & 619 & 1168 & 1205 & 875 \\
\hline ft & 0,033 & 0,041 & 0,044 & 0,038 \\
\hline$\Delta$ Pt & 49245 & 14009 & 16926 & 15856 \\
\hline As & 0,0137 & 0,0148 & 0,0131 & 0,0192 \\
\hline De & 0,025 & 0,019 & 0,0149 & 0,01 \\
\hline vs & 0,47 & 0,43 & 0,495 & 0,337 \\
\hline Res & 25281 & 18327 & 15844 & 7633 \\
\hline Prs & 7,5 & 7,5 & 7,5 & 7,6 \\
\hline hs & 920 & 1034 & 1288 & 1172 \\
\hline fs & 0,315 & 0,331 & 0,337 & 0,376 \\
\hline$\Delta$ Ps & 24909 & 15717 & 21745 & 12532 \\
\hline U & 317 & 376 & 409,3 & 273 \\
\hline A & 61,5 & 52,9 & 47,5 & 69,8 \\
\hline Ci & 19007 & 17599 & 16707 & 20356 \\
\hline Co & 1304 & 440 & 523,3 & 457,2 \\
\hline Cod & 8012 & 2704 & 3215,6 & 2809 \\
\hline Ctot & 27020 & 20303 & 18822,6 & 23165,9 \\
\hline
\end{tabular}

Fuente: Los autores.

Se puede observar que el diseño obtenido utilizando el algoritmo propuesto en este trabajo presenta una mayor longitud de los tubos que los demás casos, además, el coeficiente de transferencia de calor del lado del tubo también es menor. Esto provocó una reducción del coeficiente global de transferencia de calor, llevando a un aumento en el área de transferencia. Una caída de presión del lado de la coraza, unido al aumento del área de transferencia proporciona un alto costo en el diseño del intercambiador. Se puede notar cómo la situación económica influye en el resultado óptimo de diseño. Se seleccionó un diámetro de tubo de menor tamaño y se obtuvo una longitude superior a la de los ejemplos. Esto proporciona un compromiso adecuado entre los costes de material y fabricación asociados con los tubos.

\section{3 CASO 3: : INTERCAMBIADOR DE AGUA DESTILADA - AGUA CRUDA [32]}

Un resumen de los resultados obtenidos con el modelo propuesto es dan en la Tabla 7, donde se ofrece una comparación con los resultados reportados por [10] utilizando PSO, por [8] utilizando el enfoque GA y con la solución de diseño original dada por Sinnot [32] y Kern [34].
Tabla 6. Propiedades físicas de entrada del caso 3.

\begin{tabular}{|l|c|r|r|}
\hline \multirow{2}{*}{\multicolumn{2}{|c|}{}} & \multicolumn{2}{c|}{ CASO 3 } \\
\cline { 3 - 4 } & & $\begin{array}{r}\text { Coraza } \\
\text { Agua } \\
\text { destilada }\end{array}$ & \multicolumn{1}{c|}{$\begin{array}{c}\text { Agubo } \\
\text { cruda }\end{array}$} \\
\hline Flujo másico & $m$ & 22,07 & 35,31 \\
\hline $\begin{array}{l}\text { Temperatura de } \\
\text { entrada }\end{array}$ & $T_{i}$ & 33,9 & 23,9 \\
\hline Temperatura de salida & $T_{o}$ & 29,4 & 26,7 \\
\hline Densidad del fluido & $\rho$ & 995 & 999 \\
\hline Calor específico & $C p$ & 4,18 & 4,18 \\
\hline Viscosidad del fluido & $\mu$ & 0,0008 & 0,00092 \\
\hline Conductividad térmica & $\lambda$ & 0,62 & 0,62 \\
\hline $\begin{array}{l}\text { Resistencia de } \\
\text { ensuciamiento }\end{array}$ & Rensuc & 0,00017 & 0,00017 \\
\hline
\end{tabular}

Fuente: [32]. 
Maida Bárbara Reyes Rodríguez et al./ ITEGAM-JETIA Vol.01, No 04, pp.09-18. Dezembro, 2015.

Tabla 7. Geometrías Óptimas del intercambiador de calor del caso 3 usando diferentes métodos.

\begin{tabular}{|l|r|r|r|r|}
\hline \multicolumn{1}{l|}{ L Literatura } & GA & PSO & MACO \\
\hline do & 4,88 & 1,548 & 1,45 & 1,71 \\
B & 0,013 & 0,016 & 0,0145 & 0,015 \\
Ds & 0,305 & 0,44 & 0,423 & 0,5 \\
$\mathrm{Pt}$ & 0,387 & 0,62 & 0,59 & 0,58 \\
$\mathrm{Nt}$ & 0,023 & 0,02 & 0,0181 & 0,0187 \\
$\mathrm{vt}$ & 160 & 803 & 894 & 815 \\
$\mathrm{Ret}$ & 1,76 & 0,68 & 0,74 & 0,76 \\
$\mathrm{Prt}$ & 36400 & 9487 & 9424 & 998 \\
$\mathrm{ht}$ & 6,2 & 6,2 & 6,2 & 6,2 \\
$\mathrm{ft}$ & 6558 & 6043 & 5618 & 3990 \\
$\Delta \mathrm{Pt}$ & 0,023 & 0,031 & 0,0314 & 0,0314 \\
$\mathrm{As}$ & 62814 & 3673 & 4474 & 2124 \\
$\mathrm{De}$ & 0,0236 & 0,0541 & 0,059 & 0,058 \\
$\mathrm{vs}$ & 0,013 & 0,011 & 0,0103 & 0,0106 \\
$\mathrm{Res}$ & 0,94 & 0,41 & 0,375 & 0,377 \\
$\mathrm{Prs}$ & 16200 & 8029 & 4814 & 5004 \\
$\mathrm{hs}$ & 5,4 & 5,4 & 5,4 & 5,39 \\
$\mathrm{fs}$ & 5735 & 2476 & 4088,3 & 3955,4 \\
$\Delta \mathrm{Ps}$ & 0,337 & 0,374 & 0,403 & 0,401 \\
$\mathrm{U}$ & 67,684 & 4365 & 4721 & 5391 \\
$\mathrm{~A}$ & 1471 & 1121 & 1177 & 1054 \\
$\mathrm{Ci}$ & 46,6 & 62,5 & 59,15 & 66,04 \\
$\mathrm{Co}$ & 16549 & 19163 & 18614 & 19740 \\
$\mathrm{Cod}$ & 4466 & 272 & 276 & 233 \\
$\mathrm{Ctot}$ & 27440 & 1671 & 1696 & 1435 \\
\hline & 43989 & 20834 & 20310 & 21175 \\
\hline
\end{tabular}

Fuente: Los autores.

La principal diferencia entre los resultados es en la caída de presión en los tubos, lo que eleva los costos de bombeo necesarios para el intercambiador. MACO muestra una reducción en costo anual de operación y el costo total de operación de descuento, determinado por una reducción de la caída de presión en los tubos. Una disminución en el coeficiente de transferencia en el lado del tubo trae una diminución en el coeficiente global, lo que provoca un aumento en el área de total de transferencia. Este aumento afecta el capital de inversión. El aumento en el capital de inversión da al traste con la mejora producida en el costo total de operación de descuento, provocando un costo total superior a los reportados por AG y PSO.

\section{CONCLUSIONES}

Los intercambiadores de calor son un componente integral de todos los sistemas térmicos. Sus diseños deben adaptarse bien a las aplicaciones en que se utilizan, de lo contrario sus comportamientos serán engañosos y sus costos excesivos. El diseño de intercambiadores de calor puede ser una tarea compleja y herramientas avanzadas de optimización son útiles para identificar el mejor y más barato intercambiador de calor para una demanda específica.

El presente estudio ha demostrado la aplicación con éxito de la técnica de MACO comparada con los GA y PSO para el diseño óptimo de un intercambiador de calor de tubo y coraza desde el punto de vista económico. La técnica MACO presentada es simple en concepto, de pocos parámetros y fácil para su implementación. Estas características impulsan la aplicación de las MACO particularmente en el diseño de sistemas térmicos, donde los problemas suelen ser complejos y tienen un gran número de variables y discontinuidad en la función objetivo. La capacidad de esta técnica se demuestra usando diferentes casos de estudio de la literatura y el comportamiento de los resultados se compara con los obtenidos por los investigadores anteriores.

La misma converge a un valor óptimo de la función objetivo dentro de muy pocas generaciones y esta característica revela la importancia de la MACO para la optimización de intercambiadores de calor. La técnica MACO puede ser 
Maida Bárbara Reyes Rodríguez et al./ ITEGAM-JETIA Vol.01, No 04, pp.09-18. Dezembro, 2015.

fácilmente modificada para adaptarse a la optimización de los diferentes sistemas térmicos. Existe una preferencia por los autores que han realizado trabajos de optimización por el uso de los métodos de Kern y de Bell Delaware.

\section{REFERENCIAS BIBLIOGRÁFICAS}

[1] Szargut, J. (2005). Exergy method: technical and ecological applications, WIT press.

[2] Bilgen, S. and İ. Sarıkaya (2015). "Exergy for environment, ecology and sustainable development." Renewable and Sustainable Energy Reviews 51: 1115-1131.

[3] Green, D. W. (2008). Perry's chemical engineers' handbook, McGraw-hill New York.

[4] Kakac, S., et al. (2012). Heat exchangers: selection, rating, and thermal design, CRC press.

[5] Chaudhuri, P. D., et al. (1997). "An automated approach for the optimal design of heat exchangers." Industrial \& engineering chemistry research 36(9): 3685-3693.

[6] R. Selbas, O. K., M. Reppich (2006). "A new design approach for shell-and-tube heat exchangers using genetic algorithms from economic point of view." Chemical Engineering and Processing.

[7] Özçelik, Y. (2007). "Exergetic optimization of shell and tube heat exchangers using a genetic based algorithm." Applied Thermal Engineering 27(11): 1849-1856.

[8] A.C. Caputo, P.M.P., P. (2008). "Heat exchanger design based on economic optimization." Applied Thermal Engineering .

[9] Fesanghary, M., et al. (2009). "Design optimization of shell and tube heat exchangers using global sensitivity analysis and harmony search algorithm." Applied Thermal Engineering 29(5): 1026-1031.

[10] V.K. Patel, R. V. R. (2010). "Design optimization of shelland-tube heat exchanger using particle swarm optimization technique." Applied Thermal Engineering.

[11] S. Sun, Y. L., C. Yan. (1993). "Optimization in calculation of shell-and-tube heat exchanger." International Communications in Heat and Mass Transfer.

[12] M. Reppich, J. K. (1994). "Optimal design of shell-andtube heat exchangers." Computer \& Chemical Engineer 18

[13] M. Saffar-Avval, E. D. (1995). "A general correlation for determining optimum baffle spacing for all types of shelland-tube exchangers." International Journal of Heat and Mass Transfer.

[14] H. Li, V. K. (1998). "Visualization and determination of local heat transfer coefficients in shell-and-tube heat exchangers for staggered tube arrangement by mass transfer measurements." Experimental Thermal and Fluid Science.

[15] B.K. Soltan, M. S.-A., E. Damangir. (2004). "Minimizing capital and operating costs of shell-and-tube condensers using optimum baffle spacing." Applied Thermal Engineering. [16] I. Ozkol, G. K. (2005). "Determination of the optimum geometry of the heat exchanger body via a genetic algorithm." International Journal of Heat and Mass Transfer.

[17] P.P.P.M. Lerou, T. T. V., J.F. Burger, H.J.M. Brake, H.
Rogalla. (2005). " Optimization of counter flow heat exchanger geometry through minimization of entropy generation." Cryogenics.

[18] L. Valdevit, A. P., H.A. Stone, A.G. Evans. (2006). "Optimal active cooling performance of metallic sandwich panels with prismatic cores." International Journal of Heat and Mass Transfer.

[19] R. Hilbert, G. J., R. Baron, D. Thevenin. (2006). "Multi objective shape optimization of a heat exchanger using parallel genetic algorithm." International Journal of Heat and Mass Transfer.

[20] B.V. Babu, S. A. M. (2007). "Differential evolution strategies for optimal design of shell-and-tube heat exchangers." Chemical Engineering Science.

[21] F. Pettersson, J. S. (2007). "Design of robust heat recovery systems in paper machines." Chemical Engineering and Processing.

[22] P. Wildi-Tremblay, L. G. (2007). "Minimizing shell-andtube heat exchanger cost with genetic algorithms and considering maintenance." International Journal of Energy Research.

[23] A.L.H. Costa, E. M. Q. (2008). "Design optimization of shell-and-tube heat exchanger." Applied Thermal Engineering. [24] B. Allen, L. G. (2008). "Optimal geometry and flow arrangement for minimizing the cost of shell-and-tube condensers." International Journal of Energy Research.

[25] G.N. Xie, B. S., Q.W. Wang. (2008). "Optimization of compact heat exchangers by a genetic algorithm." Applied Thermal Engineering.

[26] J.M. Ponce-Ortega, M. S.-G., A. Jimenez-Gutierrez. (2008). "Design and optimization of multipass heat exchangers." Chemical Engineering and Processing

[27] J.M. Ponce-Ortega, M. S.-G., A. Jimenez-Gutierrez. (2009).

"Use of genetic algorithms for the optimal design of shelland-tube heat exchangers." Applied Thermal Engineering.

[28] KERN, D. Q. (1997). "Procesos de Transferencia de Calor". México, Editorial Continental s.a.

[29] Fraas, A. P. (1989). Heat Exchanger Design. New York, John Wiley.

[30] M.M. Ohadi (2000). "The Engineering Handbook." CRC Press LLC.

[31] Selbaş, R., et al. (2006). "A new design approach for shelland-tube heat exchangers using genetic algorithms from economic point of view." Chemical Engineering and Processing: Process Intensification 45(4): 268-275.

[32] R.K. Sinnot, J. M. C., J.F. Richardson (1996). "Chemical Engineering Design." Butterworth-Heinemann. vol. 6.

[33] Hewitt, G. F. (1998). Heat Exchanger Design Handbook. New York, Begell House.

[34] Kern, D. Q. (1950). Process Heat Transfer. New York, McGraw-Hill.

[35] M. Taal, I. B., J. Klemes, P. Stehlik (2003). "Cost estimation and energy price forecast for economic evaluation of retrofit projects." Applied Thermal Engineering. 